Boise State University

ScholarWorks

Materials Science and Engineering Faculty

Department of Materials Science and

Publications and Presentations

Engineering

2020

\title{
Time-of-Flight Depth-Resolved Imaging with Heralded Photon Source Illumination
}

Ximing Ren

Boise State University

Publication Information

Ren, Ximing; Frick, Stefan; McMillan, Alex; Chen, Songmao; Halimi, Abderrahim; Connolly, Peter W.R.; . . . and Buller, Gerald S. (2020), "Time-of-Flight Depth-Resolved Imaging with Heralded Photon Source Illumination". Conference on Lasers and Electro-Optics, OSA Technical Digest, AM3K.6-1 - AM3K.6-2. https://dx.doi.org/10.1364/CLEO_AT.2020.AM3K.6

This document was originally published in Conference on Lasers and Electro-Optics, OSA Technical Digest by Optical Society of America (OSA) Publishing. Copyright restrictions may apply. doi: 10.1364/CLEO_AT.2020.AM3K.6 


\title{
Time-of-Flight Depth-Resolved Imaging with Heralded Photon Source Illumination
}

\author{
Ximing Ren ${ }^{1,3}$, Stefan Frick ${ }^{2,4}$, Alex McMillan², Songmao Chen ${ }^{1,5}$, Abderrahim \\ Halimi ${ }^{1}$, Peter W. R. Connolly ${ }^{1}$, Siddarth K. Joshi ${ }^{2}$, Stephen Mclaughlin ${ }^{1}$, John G. \\ Rarity $^{2}$, Jonathan C. F. Matthews ${ }^{2}$, Gerald S. Buller ${ }^{1}$ \\ 1 School of Engineering and Physical Sciences, Heriot-Watt University, Edinburgh, EH14 4AS, UK. \\ 2 Quantum Engineering Technology Labs, H. H. Wills Physics Laboratory and Department of Electrical \& \\ Electronic Engineering, University of Bristol, Bristol, BS8 1FD, UK. \\ 3 Micron School of Materials Science and Engineering, Boise State University, Boise, USA. \\ 4 Department of Experimental Physics, University of Innsbruck, Innsbruck, Austria. \\ 5 Xi'an Institute of Optics and Precision Mechanics, Chinese Academy of Science (CAS), Xi'an, China. \\ Ximing.Ren@outlook.com
}

\begin{abstract}
We demonstrate 3D time-of-flight imaging from a scattering target illuminated with a heralded single photon source. Our image reconstruction algorithm achieves millimeter depth resolution with only 0.3 average detected photons per image pixel. (C) 2020 The Author(s)
\end{abstract}

\section{Introduction}

The capability to sense and image objects at range is a rapidly growing area of interest within the field of quantum metrology. In particular, high precision depth resolved imaging has found many uses including autonomous vehicle operation and defense applications of target identification and rangefinding. Depth information of remote targets is commonly extracted optically using a LIDAR style approach, combining a pulsed probe light source with time-resolved detection of backscattered photons to measure the round trip time to an object. Typically, such systems utilise bright pulsed laser light to overcome the geometrical optical losses introduced by non-cooperative scattering targets and achieve sufficient contrast of the rangefinding signal relative to uncorrelated noise from background light and electronic detector noise. Here we demonstrate 3D direct time-of-flight imaging (DTOF) from a Lambertian scattering target illuminated with a raster-scanned beam of heralded single photons from a CW pumped, photon pair source.

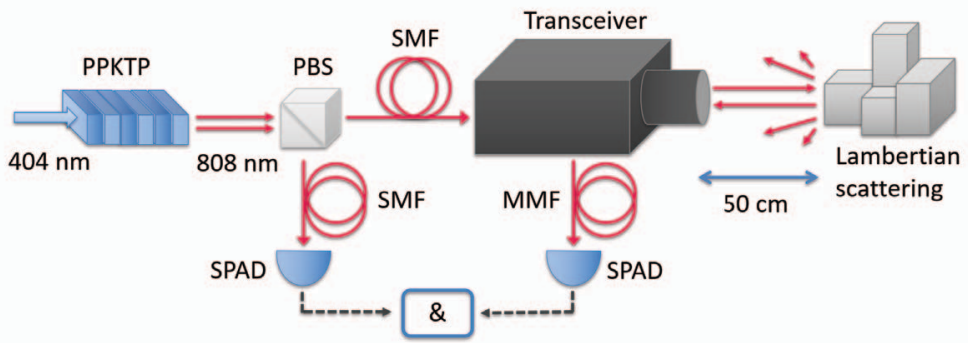

(a)

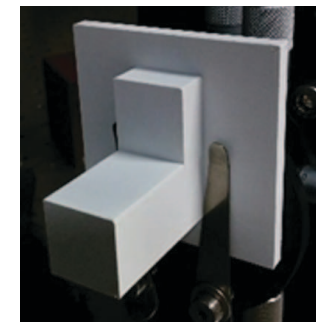

(b)

Fig. 1: (a) Simplified schematic diagram of the experimental setup. PPKTP: periodially-poled potassium titanyl phosphate crystal, PBS: polarising beam-splitter, SMF: single-mode fibre, MMF: multimode fibre, SPAD: singlephoton avalanche photodiode detector, \&: time-tagging electronics; (b) Photograph of the sample.

The experimental setup is depicted in Figure 1. Probe light for the DTOF imaging was generated via type-II parametric down-coversion in a $30 \mathrm{~mm}$ length, periodically-poled KTP crystal (Raicol) [1]. The crystal is pumped with $30 \mathrm{~mW}$ of $\mathrm{CW}$ laser light at $404 \mathrm{~nm}$ (Toptica, iBeam), generating orthogonally polarised signal and idler photon pairs at the degenerate wavelength of $808 \mathrm{~nm}$, which are subsequently split with a polarising beam-splitter and coupled to single mode fibres. Idler photons from the source were measured directly using a low dark count and low timing jitter SPAD detector (PDM series, Micro Photon Devices) providing a heralding signature and timing reference for the signal photons. A scanning transceiver system was used to focus and raster scan the signal photons across the the surface of a 3D target at a distance of $50 \mathrm{~cm}$, using computer-controlled galvanometer steering 
mirrors [2]. Backscattered photons from the target were collected back into the transceiver along a common path with the illumination light, before being coupled to a multimode fibre and detected using a second SPAD. Timetagging electronics (HydraHarp 400, PicoQuant) recorded the relative arrival time for signal and idler photon detection events, allowing the correlated coincident detection of paired photons to be identified, and variations in the round trip time for the backscattered photons due to depth features on the sample surface to be measured.

\section{Results and analysis}

A $15 \mathrm{~mm}$ x $15 \mathrm{~mm}$ area of the sample was divided into 40 x 40 pixels, which were raster scanned sequentially with a dwell time for data acquisition of $140 \mathrm{~s}$ at each pixel. A depth range of interest was predetermined by time gating, such that we post-select as coincident only two-fold counts occurring within a timing window of $400 \mathrm{ps}$. Within this window, the measured two-fold count rate was $0.14 \mathrm{~min}^{-1}$ at a measured singles count rate of $57 \mathrm{k} \mathrm{counts} \mathrm{s}^{-1}$ for the heralding idler photons. A time histogram of the relative arrival time of signal and idler photons, taken directly from the source, showed a clear coincidence peak with 40 ps full-width-half-maximum, limited by the timing jitter of the detectors. This ultimately determines the depth resolution of the imaging system.

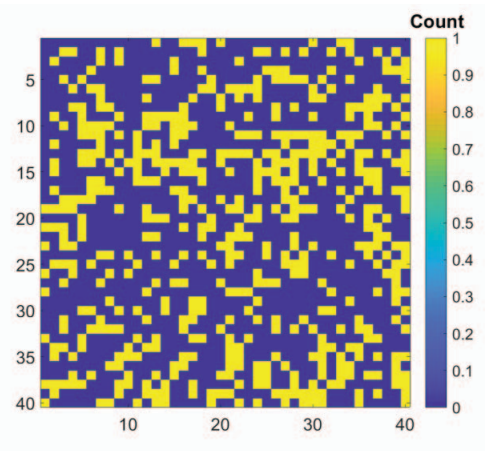

(a)

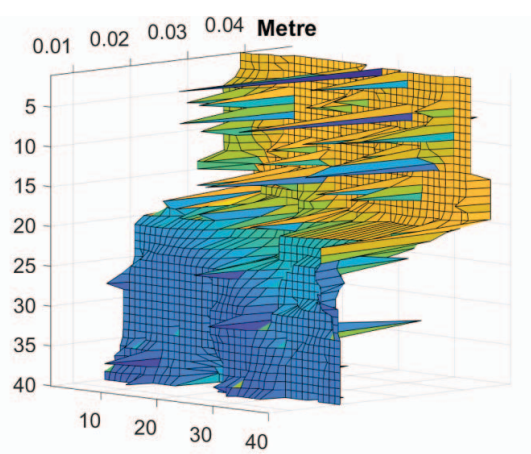

(b)

Fig. 2: (a) Raw data showing two-fold coincidence counts collected across a $40 \mathrm{x} 40$ pixel raster scan of the sample. A total of 539 two-fold coincidence events were observed across the entire image; (b) Post-processed image of the sample after applying an image reconstruction algorithm.

Due to the high optical losses involved in DTOF measurements, the total number of coincidences detected across the entire image is very low, as seen in Figure 2(a). In order to recover surface features of the sample, an image was obtained by the approach of restoration of depth and intensity using total variation (RDI-TV) [3]. Despite the sparsity of the raw data set, this approach is able to reconstruct a faithful image of the sample under test, as seen in Figure 2(b). The two well-defined and uniform planar surfaces of the sample with $30 \mathrm{~mm}$ separation in depth are clearly apparent. The precision of the depth estimation was found to be better than $3 \mathrm{~mm}$, by comparison against a ground-truth image obtained with bright light from a pulsed supercontinuum source.

To our knowledge, this is the lowest intensity illumination that has been used for DTOF imaging, and also the first time a correlated pair photon source has been used for this purpose. This proof of principle experiment paves the way for new ultra-low light level applications of TOF, in particular covert and eyesafe range imaging. The use of CW pumped photon sources already provides several advantages in this area, such as avoiding the range ambiguity that occurs with pulsed sources and matching the statistical distribution of thermal background light. Future work will look to increase both the source brightness and spectral bandwidth of the heralded photons to more closely mimic ambient light properties and reduce image acquisition time [4].

\section{References}

1. P. A. Moreau et al., "Demonstrating an absolute quantum advantage in direct absorption measurement," Scientific Reports 7, 6256 (2017).

2. A. McCarthy et al., "Long-range time-of-flight scanning sensor based on high-speed time-correlated singlephoton counting," Applied Optics 48, 6241-6251 (2009).

3. S. Chen et al., "Non-Local Restoration Of Sparse 3d Single-Photon Data," in 27th European Signal Processing Conference (EUSIPCO), pp. 1-5. IEEE, (2019).

4. S. Frick et al., "The Invisible Rangefinder," in 2017 European Conference on Lasers and Electro-Optics and European Quantum Electronics Conference, (Optical Society of America), paper CD_9_3 (2017). 